\title{
Risk stratification by anamnesis increases SARS-CoV-2 test efficiency in cancer patients
}

\author{
Christian Cornelius Arnold ${ }^{1}$ Jens von der Grün ${ }^{1,2}$ • Mark Christoph Brekner ${ }^{1}$ Jörg Licher ${ }^{1}$. \\ Emmanouil Fokas ${ }^{1,2,3}$. Claus Rödel ${ }^{1,2,3}$. Maximilian Fleischmann'
}

Received: 6 June 2021 / Accepted: 7 September 2021 / Published online: 7 October 2021

(C) The Author(s) 2021

\begin{abstract}
Purpose To evaluate the impact of testing asymptomatic cancer patients, we analyzed all tests for severe acute respiratory syndrome coronavirus-2 (SARS-CoV-2) before and during radiotherapy at a tertiary cancer center throughout the second wave of the pandemic in Germany.

Methods Results of all real-time polymerase chain reaction (RT-PCR) tests for SARS-CoV-2 performed at our radio-oncology department between 13 October 2020 and 11 March 2021 were included. Clinical data and anamnestic information at the time of testing were documented and examined for (i) the presence of COVID-19-related symptoms and (ii) virus-related anamnesis (high-risk [prior positive test or contact to a positive tested person within the last 14 days] or low-risk [inconspicuous anamnesis within the last 14 days]).

Results A total of 1056 SARS-CoV-2 tests in 543 patients were analyzed. Of those, 1015 tests were performed in asymptomatic patients and 41 tests in patients with COVID-19-associated symptoms. Two of $940(0.2 \%)$ tests in asymptomatic patients with low-risk anamnesis and three of $75(4.0 \%)$ tests in asymptomatic patients with high-risk anamnesis showed a positive result. For symptomatic patients, SARS-CoV-2 was detected in three of $36(8.3 \%)$ low-risk and three of five $(60.0 \%)$ high-risk tests.

Conclusion To the best of our knowledge, this is the first study evaluating the correlation between individual risk factors and positivity rates of SARS-CoV-2 tests in cancer patients. The data demonstrate that clinical and anamnestic assessment is a simple and effective measure to distinctly increase SARS-CoV-2 test efficiency. This might enable cancer centers to adjust test strategies in asymptomatic patients, especially when test resources are scarce.
\end{abstract}

Keywords SARS-CoV-2 testing $\cdot$ COVID-19 $\cdot$ Radiation Oncology $\cdot$ Radiotherapy $\cdot$ Real-time polymerase chain reaction (PCR) tests

\section{Introduction}

The emergence of SARS-CoV-2 has changed the world. Until May 2021, more than 3.4 million people around the globe died of the disease caused by the virus, called COVID-19 [1]. The infection is most harmful in the elderly

Availability of data and material The datasets generated and/or analyzed during the current study are available from the corresponding author on reasonable request.

$\square$ Christian Cornelius Arnold

christian.arnold@kgu.de

1 Department of Radiotherapy and Oncology, Goethe University, 60590 Frankfurt am Main, Germany

2 German Cancer Research Center (DKFZ), Heidelberg and German Cancer Consortium (DKTK), Partner Site Frankfurt am Main/Mainz, Frankfurt am Main, Germany

3 Frankfurt Cancer Institute (FCI), Goethe University, Frankfurt am Main, Germany population, but also in younger individuals with comorbidities. Cancer patients, particularly those under therapy, are suspected to be at higher risk for severe COVID-19 [2-6]. This has led to immense efforts in cancer centers to prevent the spread of the infection within departments $[7,8]$. One strategy might be the testing of asymptomatic patients before and during anticancer treatment to detect unrecognized carriers [9]. This has been recommended in guidelines by scientific organizations such as the European Society for Medical Oncology (ESMO) [10]. Yet, evidence about the impact of systematic testing in asymptomatic cancer patients is lacking $[11,12]$. For radiation oncology 
departments, regular screening of asymptomatic patients is resource consuming because radiotherapy regimens frequently have to be provided over a period of up to 2 months [13]. Hence, there is a clear need for efficient test strategies to manage the ongoing COVID-19 pandemic; the end of which cannot be foreseen $[14,15]$. In this study, we report the results of testing asymptomatic as well as symptomatic cancer patients for SARS-CoV-2 at the Radiation Oncology Department of the University Hospital Frankfurt, Germany, during the second wave of the COVID-19 pandemic in autumn/winter 2020/2021. In addition, anamnestic information at the time of testing was assessed to evaluate possible improvements of test efficiency.

\section{Methods}

This study was approved by the Ethics Committee of the Medical Faculty of Goethe University Frankfurt, Germany (vote number: UCT-24-2021).

Clinical data, treatment protocols and virus-related anamnesis were collected from all cancer patients receiving radiotherapy between 13 October 2020 and 11 March 2021. In addition, all SARS-CoV-2 tests in the stated time period were evaluated. For each test, nasal and pharyngeal respiratory swabs were taken for detecting SARS-CoV-2 RNA by real-time polymerase chain reaction (RT-PCR). All tests were performed at the Department of Virology, University Hospital Frankfurt.

Test results were documented in the clinical information system ORBIS (Agfa HealthCare) and correlated with individual clinical and anamnestic data at the time of testing, i.e., (i) presence of COVID-19 symptoms and (ii) virusrelated anamnesis (see below).

In case of a positive test result, contact tracing was performed and the clinical course of COVID-19 was documented.

Furthermore, SARS-CoV-2 infection rates in the observation period were assessed by the daily 7-day incidences within the community of Frankfurt and nationwide.

\section{Testing strategy}

Clinical and anamnestic data of all patients were evaluated daily. First, patients were examined via questionnaire or orally for symptoms associated with COVID-19 (i.e., fever, dyspnea, cough, anosmia, sore throat, rhinitis). Second, virus-related anamnesis was assessed and classified as high-risk if the patient had contact with a positive tested person or had been tested positive themselves within the last 14 days. In case of inconspicuous virus-related history, the anamnesis was classified as low-risk. All patients with high-risk anamnesis (irrespective of the exact risk factor) were considered as potentially infectious and consequences for the actual treatment (as described below) were similar.

A RT-PCR test was performed for every patient displaying suspicious symptoms. In case of negative result, repeat tests were conducted whenever clinically necessary.

All patients whose anamnesis was estimated as highrisk were tested, and testing was repeated after 5, 10, and in some cases 14 days.

Furthermore, all patients were tested before inpatient treatment (like chemotherapy, brachytherapy or supportive care) or outpatient chemotherapy at our day clinics. For hospitalized patients, who were scheduled for a transfer from another ward to our department, a recently conducted SARS-CoV-2 test $(<48 \mathrm{~h})$ was required. In addition, all patients on our ward were tested in a 7-day rhythm.

Because of increasing incidence in the whole population, we intensified our testing strategy in the time period concerned. From 13 November 2020 every patient was additionally tested before a treatment planning CT scan was done.

In case of a positive test result, tests to monitor the infection were performed at varying time intervals, depending on the treatment circumstances and clinical presentation of the individual.

\section{Safety measures within the department}

During the test period, general safety measures had been implemented into the daily routine. Medical staff members and patients were required to wear surgical or FFP2 face masks at all times. Everyone was obliged to keep physical distance of at least $1.5 \mathrm{~m}$, whenever possible. For that purpose, the seating arrangements in the waiting rooms were converted and time schedules were adapted to avoid an accumulation of patients. Whenever feasible, follow-up visits were postponed or carried out by telephone call only. Accompanying persons were allowed in exceptional cases only (e.g., need for translation). Prescreening by telephone and before entrance to our department via questionnaire or orally was strictly conducted every day to detect suspicious symptoms or anamnestic risk-factors, like those mentioned above.

If a patient was tested positive for SARS-CoV-2 before treatment initiation, radiotherapy was postponed whenever feasible. If radiotherapy was considered to be urgently needed, the patient was treated under high hygiene standards per in-house protocol at the end of the daily time schedule. In patients with high-risk virus-related anamnesis and negative test result, the same protective conditions were applied.

Whenever feasible, hospitalized patients were attended to in single rooms to minimize contacts. A visitor ban on 
our ward was instated at the beginning of the pandemic, with an exception only for patients in the terminal stage.

\section{Results}

From 13 October 2020 to 11 March 2021, 1056 RT-PCR tests for SARS-CoV-2 were performed. The total number of tested patients was 543. Table 1 shows the clinicopathological characteristics of the examined cohort. The number of tests per patient ranged from 1 to 11 (median: 1). Of 543, $203(37.4 \%)$ patients had $\geq 2$ tests. Of 1056 tests, $1015(96.1 \%)$ were carried out in patients classified as asymptomatic for COVID-19-associated symptoms and 41 of $1056(3.9 \%)$ tests were performed in patients classified as symptomatic for COVID-19-associated symptoms.

In 940 of $1015(92.6 \%)$ tests in asymptomatic patients, virus-related anamnesis was classified as low-risk. In 75 of $1015(7.4 \%)$ tests in asymptomatic patients, virus-related anamnesis was classified as high-risk. SARS-CoV-2 was detected in 5 of $1015(0.5 \%)$ asymptomatic cases. Two of $940(0.2 \%)$ tests in asymptomatic patients with low-risk anamnesis had a positive result, whereas 3 of $75(4.0 \%)$ high-risk tests were positive. The positivity rate-ratio between asymptomatic high-risk tests and asymptomatic lowrisk tests was $20(4 / 0.2)$.

Of 41 tests, $36(87.8 \%)$ were performed in symptomatic patients with low-risk anamnesis and 5 of 41 (12.2\%) tests in symptomatic patients with high-risk anamnesis. Six of $41(14.6 \%)$ symptomatic tests revealed a positive result. SARS-CoV-2 was detected in 3 of $36(8.3 \%)$ symptomatic low-risk tests and 3 of $5(60.0 \%)$ symptomatic high-risk tests. The positivity rate ratio between symptomatic highrisk tests and symptomatic low-risk tests was $7.2(60 / 8.3)$. Table 2 gives an overview of the test results and clinical/ anamnestic information at the time of testing. Additional data on positivity rates in the patient cohort with high-risk anamnesis are listed in Supplementary Table 1.

Total number of positive tested patients was 8 . Three of $8(37.5 \%)$ patients were tested positive twice. Six of $8(75 \%)$ patients were tested positive before treatment initiation and treatment was postponed for 10-40 days (median: 24.5 days), until the patient was tested negative. Two of $8(25 \%)$ patients were tested positive during treatment and, because of mild symptoms, radiotherapy could be continued without noteworthy interruption (0 and 1 days).

All 4 patients who were tested positive without initially having suspicious symptoms developed mild to moderate symptoms within a few days. No serious clinical course of COVID-19 was recorded. No case of infection was traced back to a source within our department. Characteristics of positive tested patients and details on clinical course as well
Table 1 Patient characteristics

\begin{tabular}{|c|c|c|}
\hline Patient characteristics & Number & Percentage \\
\hline Total & 543 & 100 \\
\hline \multicolumn{3}{|l|}{ Gender } \\
\hline Male & 291 & 53.6 \\
\hline Female & 252 & 46.4 \\
\hline $\begin{array}{l}\text { Age at radiotherapy onset, median } \\
\text { (range, years) }\end{array}$ & $65(4-93)$ & - \\
\hline \multicolumn{3}{|c|}{ Karnofsky Performance Status at radiotherapy onset } \\
\hline $100-90$ & 283 & 52.1 \\
\hline $80-70$ & 171 & 31.5 \\
\hline $60-50$ & 74 & 13.6 \\
\hline $40-30$ & 15 & 2.8 \\
\hline $\begin{array}{l}\text { BMI at radiotherapy onset, median } \\
\text { (range) }\left[\mathrm{kg} / \mathrm{m}^{2}\right], n=488\end{array}$ & $\begin{array}{l}24.7 \\
(14.8-64.4)\end{array}$ & - \\
\hline$<18.5$ & 30 & 6.1 \\
\hline $18.5-30$ & 378 & 77.5 \\
\hline$\geq 30$ & 80 & 16.4 \\
\hline \multicolumn{3}{|l|}{ Tumor entity } \\
\hline Brain & 42 & 7.7 \\
\hline Head and neck & 78 & 14.4 \\
\hline Breast & 80 & 14.7 \\
\hline Lung & 64 & 11.8 \\
\hline Upper gastrointestinal tract & 21 & 3.9 \\
\hline Lower gastrointestinal tract & 35 & 6.4 \\
\hline Prostate & 75 & 13.8 \\
\hline Other urological & 9 & 1.7 \\
\hline Gynecological & 34 & 6.3 \\
\hline Dermatological & 27 & 5.0 \\
\hline Hematological & 55 & 10.1 \\
\hline Sarcoma & 12 & 2.2 \\
\hline Other & 11 & 2.0 \\
\hline \multicolumn{3}{|l|}{ Tumor stage } \\
\hline Localized disease & 383 & 70.5 \\
\hline Metastatic disease & 160 & 29.5 \\
\hline \multicolumn{3}{|l|}{ Treatment intention of radiotherapy } \\
\hline Curative (definitive) & 193 & 35.5 \\
\hline Curative (neoadjuvant/adjuvant) & 181 & 33.3 \\
\hline Palliative & 169 & 31.1 \\
\hline \multicolumn{3}{|l|}{ Concomitant systemic therapy } \\
\hline None & 416 & 76.6 \\
\hline Chemotherapy & 122 & 22.5 \\
\hline Immunotherapy & 3 & 0.5 \\
\hline Chemotherapy + immunotherapy & 2 & 0.4 \\
\hline $\begin{array}{l}\text { Number of radiotherapy fractions }{ }^{\mathrm{a}} \text {, } \\
\text { median (range) }\end{array}$ & $15(1-44)$ & - \\
\hline $\begin{array}{l}\text { Treatment duration }{ }^{\mathrm{a}} \text {, median (range, } \\
\text { days) }\end{array}$ & $22(1-80)$ & - \\
\hline
\end{tabular}

$B M I$ Body mass index

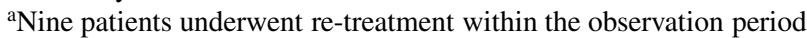


Table 2 Test results and clinical/anamnestic information at the time of testing

Positive tests/Number of tests (positivity rate)

Total: 11/1056 (1.0\%)

Patients without COVID-19associated symptoms $5 / 1015(0.5 \%)$

\begin{tabular}{llll} 
Anamnestic & $\begin{array}{l}\text { Anamnestic } \\
\text { low-risk }^{\mathrm{a}}\end{array}$ & $\begin{array}{l}\text { Anamnestic } \\
\text { high }^{\mathrm{b}}\end{array}$ & $\begin{array}{l}\text { Anamnestic } \\
\text { lowisk }^{\mathrm{a}}\end{array}$ \\
2/940 $(0.2 \%)$ & $3 / 75(4.0 \%)$ & $3 / 36(8.3 \%)$ & $3 / 5(60.0 \%)$ \\
\hline
\end{tabular}

a.e., no prior positive test and no contact to a positive tested person within the last 14 days before testing

bi.e., prior positive test or contact to a positive tested person within the last 14 days before testing

as implications for radiotherapy are shown in Supplementary Table 2 .

Local and national 7-day incidence rates were both high within the observation period, with a higher mean 7-day incidence rate locally (139.7 vs 112.9 ). Fig. 1 portrays the local and national 7-day incidence rates over time.

\section{Discussion}

Our study shows a relatively low prevalence of asymptomatic SARS-CoV-2 infections in a large cohort of cancer patients before and during radiotherapy although incidence rates in the local and national population were high within the observation period. The data also reveal that efficiency of testing can be multiplied when individual risk factors of virus-related anamnesis are taken into account. The prevalence of asymptomatic carrying of SARS-CoV-2 in cancer patients has been reported before but, to the best of our knowledge, this is the first study distinguishing positivity rates by individual anamnestic risk-factors.

Regarding previous studies, the prevalence in the cohort of cancer patients seems to be lower or at least not higher than in the whole population [16-19]. Early data from Marschner et al. were at a similar low level compared to our results and showed a positivity rate of $0.72 \%$ in asymptomatic cancer patients before initiation of radiotherapy [20]. These findings are in line with the data of Prabhu et al., who reported their systematic testing results before and during radiotherapy with SARS-CoV-2-positivity rates of $0.7 \%$ and $0.3 \%$, respectively [21]. Modi et al. recently reported their results of preradiotherapy testing in four US institutes and also found low prevalences (0.4-2.6\%) in asymptomatic patients despite a high incidence of cases in the area. The authors concluded that future test strategies may focus on risk stratification [22].

Since there is no consensus on the utility of systematic testing in cancer patients under active treatment [18-23], increasing test efficiency by risk stratification might indeed be desirable. The rational first step of risk stratification should clearly be the clinical assessment of patients. Unsurprisingly, in our study, positivity rates of patients classified as symptomatic were much higher than in the asymptomatic cohort and the utility of testing symptomatic patients is unquestionable. Nevertheless, our data reveal that risk stratification by virus-related anamnesis might increase test efficiency, also in asymptomatic patients, by a multiple. The positivity rate in asymptomatic patients with highrisk anamnesis, was much higher than in the asymptomatic low-risk cohort. Plus, only a fraction of the total test count
Fig. 1 Local and national 7-day incidence rates in the observation period and dates of patients' initial positive test for SARS$\mathrm{CoV}-2$. Dates on $x$-axis given as day.month.year



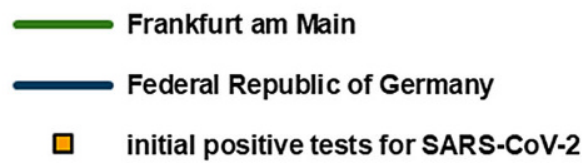


was performed in the high-risk cohort. This highlights the usefulness of a simple daily assessment of virus-related anamnesis and suspicious symptoms and could enable care providers to select patients efficiently before testing, especially when test capacities are confined. The reverse is also true: conducting consequent, daily anamnesis can trigger rational, well-chosen tests. This might be a crucial instrument to detect asymptomatic carriers and to improve avoidance of uncontrolled viral spread.

Future test strategies for patients should not only take rigid parameters into account, but should also consider the individual treatment settings. Even though recent studies indicate that continuing radiotherapy and even systemic therapies in cancer patients with (mild) COVID-19 is feasible [24-29], testing for SARS-CoV-2 before every systemic therapy cycle could be a useful measure to prevent treatment complications [7, 30]. Inpatient treatment significantly multiplies the transmission possibilities of asymptomatic carriers and the cohort of hospitalized patients might be particularly vulnerable for severe COVID-19. Therefore, testing before hospital admission and additionally in long-stay patients (e.g., in a 7-day rhythm) certainly have value. On the other hand, most (radio)oncological patients are treated partly or completely in an outpatient setting.

A consistent approach to detect every single (asymptomatic) SARS-CoV-2 infection would require an enormous use of time and resources in terms of medical staff, testing material and personal equipment. In fact, most of our outpatients were only tested once, before treatment initiation. However, not a single transmission within our center was observed (the formation of an asymptomatic cluster does not seem probable). This indicates that the consistent execution of general safety/hygienic ensured feasibility and safety of anticancer treatment in our department. Hence, especially in outpatient settings, the presented risk stratification by clinical and anamnestic assessment could be of great help, allowing the test strategy to be adjusted rationally to save resources but without compromising the safety of treatments.

The overall large sample size in our study might strengthen our considerations, but definitive conclusions for future clinical practice must be drawn carefully. The most obvious limitations of this study are the retrospective design and the restriction to a single center patient cohort. To validate our classification system of low-risk and highrisk anamnesis, prospective (multicentric) trials would have to be conducted. Even though RT-PCR is established as gold standard for the detection of SARS-CoV-2, falsenegative results are a potential source of error [31]. Ongoing mutations of SARS-CoV-2 that lead to divergent pathogenic attributes might complicate the transferability of the present data to future scenarios [32]. Moreover, the presented data have to be utilized carefully, since the results and consequences for clinical practice are highly dependent on overall incidence, societal resources and general politics.

The ongoing vaccination of the population will hopefully be the decisive game changer in everyday practice, enabling cancer centers to de-escalate various safety measures. However, for now, robust data for (long-term) vaccine efficacy for the purpose of COVID-19 prevention and reduction of virus transmittability in cancer patients is lacking [33]. As mentioned above, mutations of SARS-CoV-2 will remain a big challenge [34]. Therefore, well-considered strategies to manage the ongoing pandemic and its subsequent waves are needed. The present study might give an indication of the direction to be taken, while noting the need for more data to ensure safe treatment settings for our patients.

\section{Conclusion}

We evaluated the correlation between individual risk factors and positivity rates of SARS-CoV-2 tests in cancer patients. Our data demonstrate that simple clinical and anamnestic assessment can distinctly increase SARS-CoV-2 test efficiency. This might enable cancer centers to adjust their test strategies, especially when test capacities are confined.

Supplementary Information The online version of this article (https:// doi.org/10.1007/s00066-021-01853-7) contains supplementary material, which is available to authorized users.

Funding Open Access funding enabled and organized by Projekt DEAL.

\section{Declarations}

Conflict of interest C. C. Arnold, J. von der Grün, M. C. Brekner, J. Licher, E. Fokas, C. Rödel and M. Fleischmann declare that they have no competing interests.

Ethical standards All procedures performed in studies involving human participants or on human tissue were in accordance with the ethical standards of the institutional (Ethics Committee of the Medical Faculty of Goethe University Frankfurt, Germany [vote number: UCT-24-2021]) and/or national research committee and with the 1975 Helsinki declaration and its later amendments or comparable ethical standards. Informed consent was obtained from all individual participants included in the study.

Open Access This article is licensed under a Creative Commons Attribution 4.0 International License, which permits use, sharing, adaptation, distribution and reproduction in any medium or format, as long as you give appropriate credit to the original author(s) and the source, provide a link to the Creative Commons licence, and indicate if changes were made. The images or other third party material in this article are included in the article's Creative Commons licence, unless indicated otherwise in a credit line to the material. If material is not included in the article's Creative Commons licence and your intended use is not permitted by statutory regulation or exceeds the permitted use, you will need to obtain permission directly from the copyright holder. To view 
a copy of this licence, visit http://creativecommons.org/licenses/by/4. $0 \%$.

\section{References}

1. Center for Systems Science and Engineering (CSSE) at Johns Hopkins University (JHU) COVID-19 dashboard. https://coronavirus. jhu.edu/map.html. Accessed 23 May 2021

2. Pinato DJ, Zambelli A, Aguilar-Company J et al (2020) Clinical portrait of the SARS-CoV-2 epidemic in European cancer patients. Cancer Discov 10:1465-1474. https://doi.org/10.1158/2159-8290. CD-20-0773

3. Lee LYW, Cazier J-B, Starkey T et al (2020) COVID-19 prevalence and mortality in patients with cancer and the effect of primary tumour subtype and patient demographics: a prospective cohort study. Lancet Oncol 21:1309-1316. https://doi.org/10.1016/ S1470-2045(20)30442-3

4. Guarneri V, Bassan F, Zagonel V et al (2021) Epidemiology and clinical course of severe acute respiratory syndrome coronavirus 2 infection in cancer patients in the Veneto Oncology Network: The Rete Oncologica Veneta covID19 study. Eur J Cancer 147:120-127. https://doi.org/10.1016/j.ejca.2021.01.021

5. Saini KS, Tagliamento M, Lambertini M et al (2020) Mortality in patients with cancer and coronavirus disease 2019: a systematic review and pooled analysis of 52 studies. Eur J Cancer 139:43-50. https://doi.org/10.1016/j.ejca.2020.08.011

6. Dai M, Liu D, Liu M et al (2020) Patients with cancer appear more vulnerable to SARS-CoV-2: a multicenter study during the COVID-19 outbreak. Cancer Discov 10:783-791. https://doi.org/ 10.1158/2159-8290.CD-20-0422

7. Al-Shamsi HO, Alhazzani W, Alhuraiji A et al (2020) A practical approach to the management of cancer patients during the novel Coronavirus disease 2019 (COVID-19) pandemic: an international collaborative group. Oncologist 25:e936-e945. https://doi.org/10. 1634/theoncologist.2020-0213

8. Matuschek C, Fischer JC, Combs SE et al (2020) Measures of infection prevention and incidence of SARS-CoV-2 infections in cancer patients undergoing radiotherapy in Germany, Austria and Switzerland. Strahlenther Onkol 196:1068-1079. https://doi.org/10.1007/ s00066-020-01681-1

9. Madariaga A, McMullen M, Sheikh S et al (2020) COVID-19 testing in patients with cancer: does one size fit all? Clin Cancer Res 26:4737-4742. https://doi.org/10.1158/1078-0432.CCR-20-2224

10. European Society for Medical Oncology (ESMO) COVID-19 adapted recommendations slide sets-General recommendations. https://www.esmo.org/guidelines/covid-19-adapted-recommenda tions-slide-sets. Accessed 27 May 2021

11. Qiu X, Miller JC, MacFadden DR, Hanage WP (2021) Evaluating the contributions of strategies to prevent SARS-CoV-2 transmission in the healthcare setting: a modelling study. BMJ Open 11:e44644. https://doi.org/10.1136/bmjopen-2020-044644

12. Haradaa G, Antonacio FF, Gongora AB et al (2020) SARS-CoV-2 testing for asymptomatic adult cancer patients before initiating systemic treatments: a systematic review. ecancer 14:1100. https://doi. org/10.3332/ecancer.2020.1100

13. Akuamoa-Boateng D, Wegen S, Ferdinandus J et al (2020) Managing patient flows in radiation oncology during the COVID-19 pandemic: reworking existing treatment designs to prevent infections at a German hot spot area University Hospital. Strahlenther Onkol 196:1080-1085. https://doi.org/10.1007/s00066-020-01698-6

14. Skittrall JP, Fortune MD, Jalal $\mathrm{H}$ et al (2021) Diagnostic tool or screening programme? Asymptomatic testing for SARS-CoV-2 needs clear goals and protocols. Lancet Reg Health - Eur 1:100002. https://doi.org/10.1016/j.lanepe.2020.100002
15. Fontanet A, Autran B, Lina B et al (2021) SARS-CoV-2 variants and ending the COVID-19 pandemic. Lancet 397:952-954. https:// doi.org/10.1016/S0140-6736(21)00370-6

16. Shah MA, Mayer S, Emlen F et al (2020) Clinical screening for COVID-19 in asymptomatic patients with cancer. JAMA Netw Open 3:e2023121. https://doi.org/10.1001/jamanetworkopen.2020. 23121

17. Johannesen TB, Smeland S, Aaserud S et al (2021) COVID-19 in cancer patients, risk factors for disease and adverse outcome, a population-based study from Norway. Front Oncol 11:652535. https:// doi.org/10.3389/fonc. 2021.652535

18. Meti N, Tahmasebi H, Leahey A et al (2021) SARS-CoV-2 testing for asymptomatic patients with cancer prior and during treatment: a single centre experience. Curr Oncol 28:278-282. https://doi.org/ 10.3390/curroncol28010032

19. Berghoff AS, Gansterer M, Bathke AC et al (2020) SARS-CoV-2 testing in patients with cancer treated at a tertiary care hospital during the COVID-19 pandemic. J Clin Oncol 38:3547-3554. https:// doi.org/10.1200/JCO.20.01442

20. Marschner S, Corradini S, Rauch J et al (2020) SARS-CoV-2 prevalence in an asymptomatic cancer cohort-Results and consequences for clinical routine. Radiat Oncol 15:165. https://doi.org/ 10.1186/s13014-020-01609-0

21. Prabhu RS, Dhakal R, Hicks AS et al (2021) Implementation, adherence, and results of systematic SARS-CoV-2 testing for asymptomatic patients treated at a tertiary care regional radiation oncology network. Radiat Oncol 16:28. https://doi.org/10.1186/s13014021-01760-2

22. Modi C, Dragun AE, Henson CF et al (2021) A statewide multiinstitutional study of asymptomatic pre-treatment testing of radiation therapy patients for SARS-CoV-2 in a high-incidence region of the United States. Adv Radiat Oncol 6:100704. https://doi.org/ 10.1016/j.adro.2021.100704

23. Cavanna L, Citterio C, Di Nunzio C et al (2021) Prevalence of COVID-19 infection in asymptomatic cancer patients in a district with high prevalence of SARS-CoV-2 in Italy. Cureus 13:e13774. https://doi.org/10.7759/cureus. 13774

24. Liu H, Yang D, Chen X et al (2021) The effect of anticancer treatment on cancer patients with COVID-19: a systematic review and meta-analysis. Cancer Med 10:1043-1056. https://doi.org/10.1002/ cam4.3692

25. Garassino MC, Whisenant JG, Huang L-C et al (2020) COVID19 in patients with thoracic malignancies (TERAVOLT): first results of an international, registry-based, cohort study. Lancet Oncol 21:914-922. https://doi.org/10.1016/S1470-2045(20)30314-4

26. Lee LY, Cazier J-B, Angelis V et al (2020) COVID-19 mortality in patients with cancer on chemotherapy or other anticancer treatments: a prospective cohort study. Lancet 395:1919-1926. https:// doi.org/10.1016/S0140-6736(20)31173-9

27. Nichetti F, Bini M, Ambrosini M et al (2020) COVID-19 risk for patients undergoing anticancer treatment at the outpatient clinic of the National Cancer Institute of Milan: the COVINT study. ESMO Open. https://doi.org/10.1136/esmoopen-2020-000883

28. Angelis V, Tippu Z, Joshi K et al (2020) Defining the true impact of coronavirus disease 2019 in the at-risk population of patients with cancer. Eur J Cancer 136:99-106. https://doi.org/10.1016/j. ejca.2020.06.027

29. Hempel L, Piehler A, Pfaffl MW et al (2020) SARS-CoV-2 infections in cancer outpatients-most infected patients are asymptomatic carriers without impact on chemotherapy. Cancer Med 9:8020-8028. https://doi.org/10.1002/cam4.3435

30. von Lilienfeld-Toal M, Giesen N, Greinix H et al (2021) Coronavirus-Infektion (COVID-19) bei Patient*innen mit Blut- und Krebserkrankungen. onkopedia leitlinien. https://www.onkopedia. com/de/onkopedia/guidelines/coronavirus-infektion-covid-19bei-patient-innen-mit-blut-und-krebserkrankungen/@ @ guideline/ html/index.html. Accessed 27 May 2021 
31. Arevalo-Rodriguez I, Buitrago-Garcia D, Simancas-Racines D et al (2020) False-negative results of initial RT-PCR assays for COVID19: a systematic review. PLoS ONE 15:e242958. https://doi.org/10. 1371/journal.pone.0242958

32. Abdool Karim SS, de Oliveira T (2021) New SARS-CoV-2 variants-Clinical, public health, and vaccine implications. N Engl J Med 384:1866-1868. https://doi.org/10.1056/NEJMc2100362
33. Monin L, Laing AG, Muñoz-Ruiz M et al (2021) Safety and immunogenicity of one versus two doses of the COVID-19 vaccine BNT162b2 for patients with cancer: interim analysis of a prospective observational study. Lancet Oncol 22:765-778. https://doi.org/ 10.1016/S1470-2045(21)00213-8

34. Neuzil KM (2021) Interplay between emerging SARS-CoV-2 variants and pandemic control. N Engl J Med 384:1952-1954. https:// doi.org/10.1056/NEJMe2103931 